BULLETIN Bulletin hispanique

HISPANIQUE Université Michel de Montaigne Bordeaux

117-2 | 2015

Métamorphose(s) : représentations et réécritures

\title{
Réécriture et métamorphose dans le Polyphème de Góngora
}

\section{Nadine Ly}

\section{(2) OpenEdition}

\section{Journals}

\section{Édition électronique}

URL : http://journals.openedition.org/bulletinhispanique/4146

DOI : 10.4000/bulletinhispanique.4146

ISSN : 1775-3821

Éditeur

Presses universitaires de Bordeaux

\section{Édition imprimée}

Date de publication : 15 décembre 2015

Pagination : 741-758

ISBN : 979-10-300-0041-2

ISSN : 0007-4640

Référence électronique

Nadine Ly, « Réécriture et métamorphose dans le Polyphème de Góngora », Bulletin hispanique [En ligne], 117-2 | 2015, mis en ligne le 15 décembre 2018, consulté le 20 avril 2019. URL : http:// journals.openedition.org/bulletinhispanique/4146; DOI : 10.4000/bulletinhispanique.4146 


\title{
Réécriture et métamorphose dans le Polyphème de Góngora
}

\author{
NADINE LY \\ Université Bordeaux Montaigne
}

La Fable de Polyphème et Galatée de Góngora permet de mettre en relief les métamorphoses littéraires de Polyphème et un double travail de réécriture : la réécriture, par le poète, des sources du poème et de leurs multiples réécritures; la réécriture, dévolue à Polyphème et ici revisitée, de certains fragments de la fable, c'est-à-dire la réécriture, toujours métamorphosée, de Góngora par lui-même.

Mots-clés : Gongora, Fable de Polyphème et Galatée, métamorphose, réécriture, tradition polyphémique.

Permite recalcar la Fábula de Polifemo y Galatea de Góngora las metamorfosis literarias de Polifemo así como un doble trabajo de reescritura: la reescritura, por el poeta, de las fuentes del poema y sus múltiples reescrituras; la reescritura, a cargo de Polifemo y vuelta a analizar aquí, de ciertos fragmentos de la fábula, es decir la reescritura, siempre metamorfoseada, de Góngora por Góngora.

Palabras claves: Góngora, Fábula de Polifemo y Galatea, metamorfosis, reescritura, tradición polifémica.

Góngora's «Fable of Polyphemus and Galatea» allows us to bring out the literary metamorphosis of Polyphemus, as well as a double work of rewriting : the poet's rewriting of the sources of the poem, of their multiple rewritings. The rewriting -allotted to Polyphemus and revisited- of certain pieces of the fable, is Góngora's rewriting, under constant metamorphosis, of himself.

Keywords: Gongora, Fable of Polyphemus and Galatea, metamorphosis, rewriting, Polyphemic tradition. 


\section{La métamorphose d'Acis dans le Polyphème DE Góngora ET La CONTAMINATIO}

La Fábula de Polifemo y Galatea de Góngora s'achève, on le sait, par la métamorphose en fleuve d'Acis, écrasé par le rocher que Polyphème, fou de douleur et de jalousie, lance sur le jeune homme après avoir surpris la fuite des amants : il s'agit là de la seule métamorphose stricto sensu repérable dans la "fábula "et incontestablement reliée non seulement au poème ovidien mais au réseau tentaculaire qu'il forme avec ses réécritures et ses traductions. Il est manifeste que la métamorphose d'Acis semble intéresser moins Góngora que l'extraordinaire scène de séduction qui culmine avec le rideau de fleurs tiré sur son union charnelle avec Galatée (octaves 23 à 42). Elle l'intéresse moins qu'elle n'a retenu l'attention d'Ovide - inventeur du personnage d'Acis dans la tradition polyphémique - comme le signale José María Micó dans El Polifemo de Luis de Góngora ${ }^{1}$ et comme le montre le récit qu'en fait Galatée à Scylla dans le poème latin :

Sous le roc qui l'avait écrasé, le sang coulait en flots de pourpre. Et d'abord, sa couleur commence à s'effacer ; c'est comme l'eau d'un fleuve troublée par un orage ; peu à peu c'est une source pure et limpide. Alors la pierre s'entrouvre : de ses flancs surgit la tige vigoureuse de verts roseaux. Le flot s'ouvre et s'échappe en bondissant du creux du rocher. Tout à coup, chose merveilleuse ! s'élève du milieu des eaux le buste du jeune homme : des cornes arment son front couronné de joncs flexibles. C'était Acis, mais plus grand, mais avec un teint verdâtre, c'était Acis changé en fleuve et ces eaux ont conservé son nom².

Dans la gigantesque entreprise qu'il mène pour déterminer le plus complètement possible Las fuentes y los temas del Polifemo de Góngora à à propos de chacune de ses 63 octaves, de chacun de ses 504 hendécasyllabes et de presque tous ses mots (la réécriture concernant les personnages, les épisodes, les thèmes et les motifs mais aussi les mots pour les dire), Antonio Vilanova dénombre, outre la source ovidienne, pas moins de 42 sources possibles, y compris une octave antérieure du même Polyphème, pour les dix vers conclusifs consacrés à la métamorphose :

1. José María Micó, El Polifemo de Luis de Góngora. Ensayo de crítica e historia literaria, Barcelona, Ediciones Península, 2001, p. 100-101 : "La misma concisión caracteriza a los vv. 495-496, que, enlazados por otro violento hipérbaton, dan la versión más escueta posible de la metamorfosis, amplificada en la octava final (pero en cualquier caso muy lejos del pormenor y de las precisiones cromáticas de Ovidio, vv. 887-897) [...] ».

2. Traduction de Louis Puget, Théodore Guiard, Chevriau et Fouquier (1876) revue par Anne Videau, présentation et annotations d'Anne Videau, LGF, coll. Classiques de Poche, 2010, 604 p., p. $465-466$.

3. Antonio Vilanova, Las fuentes y los temas del Polifemo de Góngora, 2 vols., Madrid, Revista de Filología española, Anejo LXVI, 1957. Cette remarquable thèse avait obtenu, en 1951, le Premio Extraordinario de Doctorado et le Premio Menéndez Pelayo. 
62.

63 y el peñasco duro

la sangre que exprimió cristal fue puro.

Je renvoie à l'ouvrage de Vilanova ${ }^{5}$ pour le détail des fragments ou des mots sous influence, soumis à la "contaminatio ", mais il n'est pas oiseux de signaler qu’à ces 42 « sources » correspond une liste de 20 auteurs cités 6 . Seul le verbe

\footnotetext{
4. L'édition de référence est : Luis de Góngora, Fábula de Polifemo y Galatea, ed. de Jesús Ponce Cárdenas, Madrid, Cátedra, n 658, 2010.

5. Pour ce qui est de l'Espagne, un an après que Vilanova eut soutenu sa thèse, José María de Cossío publiait ses tout aussi classiques Fábulas mitológicas en España, Madrid, EspasaCalpe, 1952, préfacées par Dámaso Alonso, dans lesquelles il se proposait - ce sont les premiers mots de son introduction - "de explorar[se] en este libro toda una provincia de nuestra geografía literaria, desasistida de esclarecimientos y, en gran parte, ignorada". Il recense 14 textes espagnols liés à Polyphème, auxquels il conviendrait d'ajouter le Canto de Polifemo a la linda Galatea de Cristóbal de Castillejo (Cristóbal de Castillejo, Fábulas mitológicas, ed. Blanca Perinán, ViareggioLuca, Mauro Baroni Editore, 1999, p. 91-101) et le récit d'Ulysse dans La Circe de Lope de Vega (Lope de Vega, La Circe, con otras rimas y prosas (1624), in Obras poéticas, ed. José Manuel Blecua, Barcelona, 1983, Canto segundo, Prosigue Ulises su relación con los amores de Polifemo y Galatea, y lo que le sucedió hasta que salió de la isla), notamment les v. 113-392, p. 976-984, dans lesquels Ulysse raconte l'épisode à Circé, la magicienne des métamorphoses, et déploie les beautés de la métamorphose que sa propre écriture impose aux diverses réécritures de la fable ovidienne, non sans emprunter aux Soledades gongorines.

Voici ces textes, les indications entre crochets, ajoutées à celles de Cossío, sont de mon fait : Andosilla Larramendi (Juan), Paráfrasis [del canto de Polifemo, publicada entre las Rimas y Prosas de Gabriel Bocángel, 1627] - Anónimo, Fiesta armónica - Barrios (Miguel de), Romance burlesco, [A Polifemo y Galatea, in Flor de Apolo, 1665] - Bernaldo de Quirós (Francisco), Romance burlesco [Fábula de Polifemo, Obras, 1656] - Carrillo y Sotomayor (Luis), [Fábula de Acis y Galatea, 1613] - Castillo Solórzano (Alonso de), Burlesca [De Polifemo, a la Academia, in Donaires del Parnaso, 1624 et 1625] - Corral (Gabriel del) [En La Cintia de Aranjuez, 1629] - Góngora - Lasso de la Vega (Gabriel) [Otro romance, in Manojuelo de romances nuevos y otras obras, 1601 : suit les traductions d'Ovide de Castillejo, Alonso Pérez, Gálvez de Montalvo et peut-être l'original latin] - López de Vega (Antonio), Romance [in Lirica poesía, 1620] - Nieto de Molina (Francisco), Burlesca [El Polifemo, in El fabulero, 1764]- Pérez (Alonso) [trad. d'Ovide : nuevo canto de Gosforosto/Polifemo, in Parte primera y segunda de La Diana de Jorge de Montemayor, 1602] Sánchez Portolés (Antonio), Romance burlesco [fábula jocosa breve de Polifemo y Galatea, in El entretenido, 1673]- Valle y Caviedes (Juan del), Romance burlesco. Bibl. Nac. Ms 17.494, folio 316 v. [Fábula de Polifemo y Galatea qui ajoute l'épisode de l'Odyssée, entre 1681 et 1692].

6. Ovide, les traductions de Andrea dell'Anguillara, de Pedro Sánchez de Viana et de Antonio Pérez Sigler, la Phèdre de Sénèque (évoquée par Pellicer), Giambattista Marino et ses sonnets polyphémiques, Fernando de Herrera, Francisco de Aldana, Juan Rufo, l'Argenis de John Barclay, la fable de Luis Carrillo y Sotomayor, Acis y Galatea, Silius Italicus (Silio Itálico), Pedro de Espinosa, Alonso de Ercilla, Juan de la Cueva, Pedro de Ońa, la strophe 28 du Polyphème de
} 
"aclamar", avant-dernier mot de tout le poème, absent par ailleurs des Soledades, échappe à la toile ovidienne et intertextuelle et semble être une "invention " gongorine, puisqu'il est seulement attesté dans le Tesoro de Covarrubias (1611), contemporain de l'écriture du Polyphème. À cette très longue liste, il convient d'ajouter, à propos de "lamiendo flores ", le renvoi que fait Pedro Díaz de Rivas au De raptu Proserpinae (II, v. 103-104) de Claudien (l'un des poètes latins préférés de Góngora) et, à propos de Doris, les rapprochements avec l'Églogue X de Virgile (v. 4-5) et le De consulatu Manlii Theodori (v. 45) de Claudien encore, toutes références signalées par Jesús Ponce?.

Une première conclusion s'impose, à partir de ce seul exemple (d'autres fragments de la «fábula» peuvent convoquer des sources différentes) : à l'intérieur de la "planète " ovidienne, et en vertu de ce que Antonio Carreira appelle "poligénesis ", et qu'on connaît sous le nom de "contaminatio ", l'imitation d'imitations, se trouvent collectées, mêlées, confondues et puissamment réélaborées les molécules verbales qui gravitent dans les réécritures européennes des Métamorphoses et qui configurent l'écriture poétique, savante et sublime de Góngora dans ses grands poèmes. Dans l'Introduction à son impressionnante édition, Jesús Ponce écrit : " [...] la propuesta temática que iba a servir de puente a su revolucionaria visión del epos, lejos de ampararse en una nebulosa modernidad, contaría con el aval de una tradición milenaria " (El complejo diálogo con la tradición : "invención " gongorina y materia polifémica, p. 33). Analysant à son tour les Solitudes, Mercedes Blanco reprend, presque dans les mêmes termes ${ }^{9}$ : "Al igual que las demás obras de Góngora, las Soledades, tan profundamente originales, deben su densidad a la integración de una milenaria tradición poética latina, italiana, portuguesa y española ». À cette différence près que l'« epillium » affiche son complexe " cordon ombilical ", alors que le grand poème de 1613 l'a définitivement coupé.

Un mot de la métamorphose gongorine, cependant, a retenu mon attention, comme elle a retenu celle des commentateurs : l'adjectif " fatal», dans la formule " escollo fatal» (63.2). Le mot est le calque (sous forme d'adjectif) du neutre pluriel «fata» ('le destin') présent chez Ovide, au vers 885 : « at nos, quod fieri solum per fata licebat, / fecimus ». Dans les somptueuses notes de sa récente édition (p. 355), Jesús Ponce Cárdenas mentionne le commentaire de Pellicer, également cité par Antonio Vilanova (op. cit., II, p. 764) : "*Escollo fatal : la roca "inevitable" que le reservó el destino (P. col. 346) ", et il ajoute la glose de

Góngora, Juan de Mena, Garcilaso et Francisco de la Torre.

7. Voir Jesús Ponce Cárdenas, ed. cit., p. 356.

8. Cette tradition, la "matière polyphémique ", est remarquablement détaillée aux pages 3545. Voici les noms des poètes : Homère, Philoxène de Cythère, Théocrite, Callimaque, Philostrate, Lucien, Virgile, Ovide (inventeur d'Acis), Giovanni Gioviano Pontano, Giovan Battista Marino et Tommaso Stigliani. À ces noms italiens, s’ajoutent ceux de Angelo Poliziano, Jacopo Sannazaro ou Pietro Bembo comme le montre l'excellent article de Mélanie Bost-Fievet, "Cur fugis, Galatea? Poétique et esthétique autour des mythes de Galatée au Quattrocento ", Revue en ligne Camenae, n 9, Juin 2011, 31 p. Site officiel de l'Université Paris-Sorbonne.

9. Je renvoie globalement au tout récent et somptueux livre de Mercedes Blanco, Góngora heroico. Las Soledades y la tradición épica, Madrid, CEEH, 2012, et pour la citation à la p. 173. 
Pedro Díaz de Ribas, qui tient compte de l'acception commune de "fatal, causa de muerte y de mal fin " (DR, fol. 220 v.). "El escollo fatal " c'est donc d'abord le rocher (représenté ici dans sa version maritime) que le destin tenait préparé pour Acis et, en même temps et secondairement le rocher mortel qui lui a été fatal. L'adjectif occupe dans le vers une place clé : seul mot "agudo " du vers, il porte l'accent (le sixième de l'hendécasyllabe) métrique central, peutêtre suivi d'une imperceptible respiration, suffisante néanmoins à le mettre en relief. J'y verrais volontiers, au risque de lui donner un poids excessif et de surinterpréter le travail du poète, l'une de ces marques d'humour que la critique gongorine décèle avec bonheur dans ses poèmes héroïques, même les plus sérieux : si le rocher est inévitable par décision du destin, ne l'est-il pas aussi pour le poète qui, à sa manière, réécrit la fable et doit la mener à sa fin prescrite, fatale? L'adjectif entrerait ainsi en résonance avec l'extrême concision de la métamorphose, condensée en réalité en moins de deux vers, puis amplifiée par la strophe finale. A la fois tenu par l'argument de la fable et l'obligation d'imiter en surpassant, Góngora, prenant ses libertés avec son prestigieux modèle, fait court quand Ovide détaille : là réside peut-être la signification profonde de l'hyperbate en forme d'anacoluthe extraordinairement figurative ${ }^{10}$ qui, selon un procédé propre à Homère ${ }^{11}$ et repris par Góngora, présente dans l'ordre (chrono)logique les mots et les images qui construisent la succession des faits et les trois agents de la transformation, l'énorme pierre, le sang et l'eau cristalline : «y el peñasco duro / la sangre que exprimió cristal fue puro» (62.7-8).

Par ailleurs, il n'est pas indifférent que dans le faisceau polygénétique qui nourrit la réécriture gongorine de la métamorphose d'Acis, figure aussi l'écriture gongorine elle-même, comme l'indique Vilanova à propos de la transformation des os blancs d'Acis en "corriente plata ": "En cuanto a la metáfora gongorina corriente plata, con la que alude a la sangre y los huesos de Acis convertidos en agua, recuérdese que ya en la octava XXVIII, 1, Góngora habia llamado sonorosa plata $^{12}$ al agua de un arroyo ». C'est cependant Jesús Ponce Cárdenas qui perçoit le plus finement les échos multiples qui relient la strophe de fermeture du Polyphème à l'octave d'ouverture de la fable proprement dite, la quatrième, qui succède aux trois strophes de "dedicatoria " au Comte de Niebla :

El cotejo de imágenes, términos reiterados y sintagmas que enlazan la estancia IV y la estrofa LXIII fuerza a los lectores atentos a plantearse un posible esbozo de estructura circular, de composición en rondel. La narración se aproximaría asi a la ringkomposition de los relatos miticos antiguos (p. 355).

10. À première lecture les vers "y el peñasco duro / la sangre que exprimió cristal fue puro" (62.7-8) ont toute l'apparence d'une rupture de construction qui n'est pas sans figurer la violence du choc, de la mort et de la résurrection sous forme d'eau limpide du misérable Acis; mais à y regarder de plus près, l'anacoluthe n'est que le résultat, réussi, d'une hyperbate : *y la sangre que exprimió el peñasco duro cristal fue puro.

11. Je renvoie aux pages lumineuses que Mercedes Blanco consacre à l'Homère espagnol dans son Góngora heroico, op. cit., Chapitres VII et VIII, p. 229-298.

12. Polifemo, 28. 1 : «La ninfa, pues, la sonorosa plata / bullir sintió del arroyuelo apenas, [...] ». Vilanova, p. 771. 
À la proposition aiguë et perspicace de ce lecteur très attentif et très savant du Polyphème qu'est Jesús Ponce, je suggère d'ajouter qu'au nombre des échos poétiques multiples qui se laissent percevoir dans la fable, et qu'au lieu d'assimiler à ses "sources " il est préférable de considérer comme lymphe nourricière composite et modulable, toujours renouvelée, il est indispensable de tenir compte, à l'intérieur même de la réécriture gongorine du poème ovidien, de certains éléments réécrits de cette réécriture. Comme si Góngora inscrivait ainsi dans le profond miroir de la tradition et comme dernier maillon d'une prestigieuse théorie, le travail de sa propre réécriture. C'est autour de la figure de Polyphème, déjà soumis par la tradition antique et classique à diverses métamorphoses, que se multiplient les signes de ce travail complexe d'imitation hétéro- et auto- référentielle.

\section{LA PREMiÈre MÉTAMORPHOSE DE POLYPHÈME}

La tradition, on le sait, connaît deux Polyphème : le premier, le plus ancien, est celui de l'Odyssée, féroce et sanguinaire berger buveur de lait, enivré et endormi par le vin que lui offre Ulysse, trompé par lui et mutilé de son œil unique par un pieu monstrueux à la pointe incandescente. Après avoir, dans le chant XIII, fait intervenir la funeste prédiction de Télémus ("L'œil unique que tu as au milieu $\mathrm{du}$ front, Ulysse te le ravira $»^{13}$ ), Ovide rappelle l'épisode au chant XIV de son poème (v. 154-222). Recueilli par Énée, Achéménide, un ancien compagnon d'Ulysse oublié sur l'île après la mutilation du Cyclope, raconte comment il a réussi à échapper au monstre fou de douleur et ensanglanté :

Je vis le Cyclope arracher le sommet d'une montagne, et jeter au milieu de la mer cette masse effroyable ; je le vis encore, de ses bras gigantesques, lancer, avec la force d'une machine, d'énormes quartiers de rocs. A la vue de ces rochers, des vagues qui menaçaient de vous submerger, je pâlissais d'effroi, comme si j'avais été sur le vaisseau. Dès que la fuite vous a sauvés d'une mort affreuse, le Géant va et revient, en rugissant, sur l'Etna. Aveugle, il étend devant lui ses larges mains pour éviter les forêts; il se heurte contre les rochers, il tourne vers la mer ses bras souillés de sang, et pousse d'horribles imprécations contre les Grecs : "Oh! s'écrie-t il, si jamais le hasard ramenait sous ma main Ulysse, ou quelqu'un de ses compagnons que je puisse assouvir toute ma rage, je lui mangerais les entrailles ; je le mettrais en pièces tout vivant, je boirais son sang avec délices ; je ferais crisser ses membres broyés sous mes dents. Que je me consolerais facilement de la perte de mon oeil ! ${ }^{14}$.

Entre l'Odyssée et les deux chants ovidiens s'interpose la première métamorphose historique, millénaire, de Polyphème : celle du monstre terrifiant en monstre amoureux, à travers les fragments de la Galatea de Philoxène de Cythère, et surtout les Idylles VI et XI de Théocrite, véritable fondateur, intéressant à plus d'un titre, de la tradition bucolico-marine du Cyclope

13. Traduction de Louis Puget revue par Anne Videau, op. cit., p. 462.

14. Ibid., p. 478-479. 
amoureux : "l'antique Polyphème, quand il était amoureux de Galatée, du temps où une barbe naissante revêtait ses lèvres et ses tempes. Son amour ne s'exprimait pas par des cadeaux de pommes, de roses, de boucles de cheveux, mais par de véritables transports; et tout le reste lui semblait accessoire $»^{15}$. Ce Polyphème, jeune et émouvant, qui oppose deux mondes et leurs créatures incompatibles, son univers terrestre et bucolique et le monde marin où évolue Galatée et où il espère pouvoir entrer en apprenant à nager..., connait la laideur de certains de ses traits et la beauté de son chant : «Je sais, charmante jeune fille, pourquoi tu me fuis. C'est parce qu'un sourcil velu s'étend sur tout mon front de l'une à l'autre oreille, unique et long, parce que j'ai au front un oil unique, et qu'un nez épaté me surmonte la lèvre. N'empêche qu'en même temps, tel que je suis, j'ai au pâturage un millier de brebis [...]. Et puis, je sais jouer de la syrinx mieux qu'aucun autre Cyclope et je chante mon amour jusqu'aux dernières heures de la nuit » ${ }^{16}$. Car la poésie et le chant, « le commerce des Piérides ", sont pour Théocrite la plus efficace pharmacopée au mal d'amour. C'est à Ovide qu'il reviendra, dans l'entreprise qu'il mène de relier à des métamorphoses les éléments du paysage et de la nature, de transformer, avec l'invention d'Acis, l'amant heureux de Galatée, le Cyclope amoureux en monstre jaloux, tout en expliquant l'origine mythique du fleuve Acis.

Góngora reconstitue à sa manière l'histoire même des réécritures de la matière polyphémique qu'il retravaille et restitue dans une langue dense, héroïque ${ }^{17}$ et sublime et dans l'organisation même des motifs et des octaves de son chef d'œuvre mythologique. Alors que dans le poème latin, c'est Galatée qui raconte l'histoire, Góngora se sépare radicalement d'Ovide en ce que les deux seules " voix " qui prennent la parole dans sa fable, sont celle du narrateur poétique lui-même et, de son propre fait et à la première personne déléguée aux Piérides ${ }^{18}$ (45. $8:$ : iReferidlo, Piérides, os ruego!»), la voix de Polyphème dans ce qui partout et toujours a été le sommet des poèmes polyphémiques : le chant qu'il adresse à Galatée. C'est à Polyphème, en effet, qu'il revient d'évoquer subrepticement, comme par effraction, et dans une dynamique parfaitement vraisemblable ce que, dans la littérature classique il a été et ce que, dans la littérature contemporaine et dans le poème de Góngora, il est devenu sous l'effet de l'amour. Car l'amour qu'il porte à Galatée parvient à vaincre la barbarie du Cyclope et notamment à lui inculquer les lois sacrées de l'hospitalité. On se rappelle que, dans l'épisode homérique, Ulysse raconte, au chant IX, comment les douze compagnons qu'il avait choisis pour aller explorer la grotte solitaire de Polyphème le supplient d'emporter quelques

15. Théocrite, Idylles, Texte établi et traduit par Ph.-E. Legrand, introduit et annoté par F. Frazier, Les Belles-Lettres, CUF, Collection de Poche, 2009, 254 p.

16. Ibid.

17. Voir l'article de Jesús Ponce Cárdenas, «Polifemo y el estilo heroico. Huellas de la épica latina en el relato gongorino ", Insula 781-782, El Polifemo de Góngora : la vigencia de un clásico, enero-febrero 2012, p. 7-10.

18. Elles sont traditionnellement invoquées par les poètes quand ils abordent un fragment difficile ou épineux, voir Jesús Ponce, ed. cit., p. 316. 
fromages, des chevreaux et des agneaux, et de regagner au plus vite avec son bateau les navires qu'ils avaient laissés au large de l'île. Ulysse refuse car, ditil : « je voulais le voir et j'espérais qu'il me ferait des présents d'hospitalité. Mais son apparition ne devait pas faire le bonheur de mes compagnons ${ }^{19}$. La sauvagerie du Cyclope, en effet, qui ne connaît ni lois divines ni humaines, se manifeste autant par les scènes d'anthropophagie auxquelles il se livre que par son attitude sacrilègement inhospitalière. Dans le poème de Góngora, Polyphème rappelle d'abord, dans les quatre premiers vers de l'octave 54, les dépouilles traditionnellement exposées par d'autres chasseurs de leurs exploits cynégétiques : le gibier caractéristique de la chasse de "montería" (qui annonce celui de la Dedicatoria al Duque de Béjar à l'entrée de la première Solitude), ici le cerf et le sanglier, là l'ours et le sanglier. La tête du sanglier fait l'objet d'une métaphore hyperbolique - inspirée par les piques dressées du sanglier ovidien du Chant VIII, v. 284-286, des Métamorphoses - : les soies hérissées du groin se métamorphosent en piques helvétiques, âprement discutées par les commentateurs ${ }^{20}$. On observe que Polyphème ne mentionne pas la dépouille du lynx ${ }^{21}$, le fauve cruel à la course ailée et à la peau tachetée évoqué par Góngora à la strophe 9, puisqu'en un temps record il le transforme en une pelisse dont il se couvre et qui l'animalise et le rend plus terrifiant encore. Ses trophées sont d'une autre nature. En effet, dans les deux vers suivants, ce sont ses proies humaines que Polyphème évoque : comme font les autres chasseurs du gibier abattu, c'est de la tête du voyageur égaré que le Cyclope tueur d'hommes orne les portes de son antre. Le macabre détail - bel exemple de "contaminatio " poétique - provient d'un épisode non polyphémique de l'Enéide (VIII, v. 196197) où Virgile décrit la grotte de Cacus ${ }^{22}$. Il revient enfin au "pareado" de clôture de proposer une issue magnifique et bouleversante qui, inversant le contenu des six vers antérieurs, transforme la "cueva, de piedad desnuda", en refuge accueillant. Ce sont alors les mots d'autres poèmes de Góngora que Polyphème met en œuvre : le sonnet de 1594 (Descaminado, enfermo, peregrino, v. 7-8: " y en pastoral albergue mal cubierto / piedad halló, si no halló camino ») ou le romance de Angélica y Medoro de 1602 ("En un pastoral albergue I [...]/ mal herido y bien curado / se alberga un dichoso joven " $)^{23}$ ainsi que, par évidente anticipation, le "bienaventurado albergue " de la Soledad primera. Il est absolument remarquable que Góngora prête ici au chant de Polyphème

19. Homère, L'Odyssée, Traduction, introduction et notes par Médéric Dufour et Jeanne Raison, Paris, Flammarion, GF n ${ }^{\circ}$ 64, 1965, Chant IX, p. 133.

20. Jesús Ponce, ed. cit., p. 339.

21. "No la Trinacria en sus montañas fiera / armó de crüeldad, calzó de viento, / que redima feroz, salve ligera / su piel manchada de colores ciento: / pellico es ya la que en los bosques era / mortal horror al que con paso lento los bueyes a su albergue reducía, / pisando la dudosa luz del día. "Pour l'identification du lynx, voir Jesús Ponce, ed. cit., p. 210-212 et, du même auteur : «El enigma de la fiera : sobre la zamarra del cíclope ", in Cinco ensayos polifémicos, Málaga, Universidad de Málaga, 2009, p. 171-239.

22. José María Micó, op. cit., p. 91.

23. Luis de Góngora, Obras completas I, ed. de Antonio Carreira, Madrid, Biblioteca Castro, 2008, p. 158 et 204 . 
les mots mêmes, dejà advenus ou à venir (mais en préparation), de deux de ses plus beaux poèmes et de son chef d'œuvre, sans doute alors en gestation. L'action civilisatrice de l'amour que le Cyclope porte à la nymphe a opéré une métamorphose traditionnellement miraculeuse :

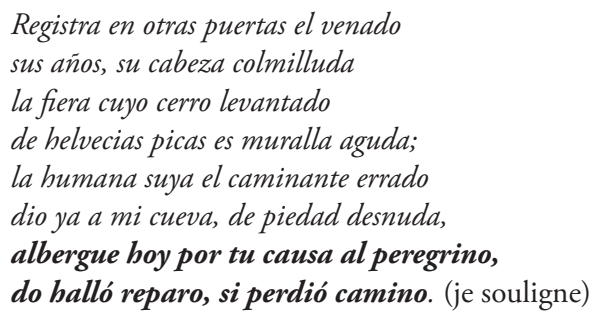

Traditionnelle, en effet, et notamment ovidienne, par la bouche de Galatée :

Ce géant farouche, l'horreur des forêts que nul n'avait pu voir impunément, le contempteur de Olympe et des dieux, sent ce que c'est que l'amour : épris de ma beauté, il brûle, il oublie son antre et ses troupeaux. Il songe à sa figure, il veut plaire : il peigne avec un râteau sa rude chevelure, il coupe avec une faux sa barbe hérissée ; il se mire dans les eaux, il compose ses traits farouches. Ce n'est plus ce géant féroce, toujours altéré de sang et affamé de meurtre : les vaisseaux abordent au rivage et le quittent sans risque ${ }^{24}$.

Or, Polyphème ne s'en tient pas là : prenant appui sur la dernière proposition d'Ovide (" les vaisseaux abordent au rivage et le quittent sans risque "), une série de quatre strophes supplémentaires (rien moins que $6 \%$ du total des octaves), parmi les plus étonnantes de la fable, relate d'abord (strophes 55-56) le naufrage d'un navire génois - alors que Polyphème, tel un nouvel Orphée, calmait les flots en imposant à la mer démontée les "dulcísimas coyundas " de son instrument, la syrinx. Brisé par les lames, le navire rejette sur la plage des coffres remplis d'épices sabéennes et de trésors de l'Inde. Dans l'octave suivante (57), le naufragé, un marchand génois, est accueilli et il trouve dans l'hospitalité de Polyphème et dans sa grotte une deuxième planche de salut. Réconforté par le régal des meilleurs fruits, il peut raconter son naufrage et il offre à son hôte un splendide cadeau d'ivoire qui, dans les quatre premiers vers de l'octave 58, fait l'objet d'une rapide "ecfrasis » : il s'agit d'un arc précieux et d'un carquois lustré, offrande d'un roi de Malacca à une divinité de Java. L'arc et le carquois permettent alors à Polyphème de s'adresser à nouveau à Galatée : si elle les accepte, elle sera "Venus del mar, Cupido de los montes". Là s'achève le chant du Cyclope.

L'intrusion dans la fable mythologique, après l'évocation des piques helvétiques, d'un navire génois moderne et uchronique, n'a pas manqué d'alerter les commentateurs. À propos de la synecdoque "ligurina haya ", Salcedo Coronel rappelle l'histoire des Ligures, peuple sauvage de l'Italie du Nord, qui ignorait tout de l'art de naviguer. Le développement de la capitale génoise,

24. Traduction de Louis Puget revue par Anne Videau, op. cit. p. 461-462. 
port de commerce et centre bancaire, ne remonte en effet qu'aux premières décades du XVII e siècle. Prenant la défense de Góngora, Salcedo invoque alors la toute puissance de la licence poétique ${ }^{25}$, mais il n'en reste pas moins que la métamorphose de Polyphème est surprenante : il illustre sa toute nouvelle générosité par un exemple qui en fait le contemporain de Góngora... C'est que le modèle, ici, n'est plus Ovide, ni Théocrite, ni aucun des Anciens, mais un poète italien moderne, Stigliani. La question avait été évoquée par Dámaso Alonso $^{26}$ qui avait découvert ce cas d'imitation gongorine dans Il Polifemo. Stanze pastorali di Tomasso Stigliani (Milan 1600), poème verbeux (dit-il) en octaves (première imitation d'Ovide en "octavas reales »), dans lequel, pour la première fois dans l'histoire poétique de Polyphème, le Cyclope offre à Galatée un bel arc, des flèches et un carquois qu'il avait arrachés à Licaspe, rescapé d'une tempête, et qu'il ne souhaite pas décrire à la nymphe, préférant qu'elle le voie elle-même. Dépassant de loin son modèle, l'imitation de Stigliani par Góngora lui vaut une pique venimeuse de Lope de Vega (je cite Dámaso Alonso) :

[...] lo cierto es que el pormenor de este arco y aljaba (inexistente, como he dicho, en Ovidio) prueba, o una fuente común y desconocida para Stigliani y Góngora, o, lo que es casi seguro, una imitación directa por Góngora de la obra del italiano. Imitación que en nada oscurece la gloria del Polifemo gongorino, ante el cual la divagadora obra de Stigliani apenas si tiene existencia estética.

$Y$ asi tal vez se explicaría aquella afirmación de Lope de Vega :

Cierto poeta de mayor esfera,
cuyo dicipulado dificulto,
de los libros de Italia fama espera:
mas porque no conozcan por insulto
los hurtos de Estillani y del Chabrera
escribe en griego, disfrazado en culto ${ }^{27}$.

La meilleure spécialiste de cette question, Giulia Poggi ${ }^{28}$ (après avoir rappelé que Samuel Guyler ${ }^{29}$ avait vu, dans les deux parties du Polyphème, la première chantée par le poète, la deuxième chantée par le Cyclope, un premier chant véritablement sublime puis son contrechant grossier, imité de Stigliani, qu'il

25. «... pero como los Poetas (según Scalígero libro 3. capit. 49. Poetic.) tienen licencia para mudar la Chronologia, y poner antes lo que fue despues, se puede satisfacer esta objeccion, pero es cosa dura que Polifemo hable con tal particularidad de la nave, que precisamente se aya de atribuyr a su tiempo, lo que no pudo ser, por las razones que hemos referido ", in El Polifemo de don Luis de Gongora comentado por don García de Salcedo Coronel [...], en Madrid, por Juan Gonçalez, Año 1629, A costa de su Autor, Reproducción facsimilar, Sevilla, ed. Extramuros, 2008.

26. Dámaso Alonso, "Los hurtos de Estillani y del Chabrera ", Obras completas, Gredos, 1982, t. VI, p. 525-539 et "Un pasaje del Polifemo imitado por Góngora ", Estudios y ensayos gongorinos, Madrid, Gredos, 1960, p. 361-364.

27. Lope de Vega, Obras sueltas, XIX, 73. Voir le sonnet 75 des Rimas humanas y divinas del Licenciado Tomé de Burguillos, ed. Antonio Carreño, Salamanque, ed. Almar, 2002, p. 244-245.

28. Giulia Poggi, «Mi voz por dulce, cuando no por mia : Polifemo entre Góngora y Stigliani », in Góngora hoy, VII. El Polifemo, coord. J. Roses, Córdoba, Diputación de Córdoba, Col. Estudios Gongorinos, 2005, p. 53-75. 
interprète comme une parodie humoristique de l'imitation poétique), préfère penser que Góngora a voulu ajouter une nouvelle voix, une voix moderne, celle de Stigliani, à toutes celles dont il tisse son grand poème et elle conclut :

Convirtiendo en genovés al Licaspe de Stigliani, y en dádiva el arco que su cíclope le roba [...], Góngora traza el esbozo de un moderno Ulises, cuyas exóticas riquezas [...] remiten a aquella politica de expansión y a aquella sed de riquezas tan duramente condenada en el epilio de la Primera Soledad. Una vez más, el Polifemo de Stigliani (quien también fue autor, entre otras cosas, de un largo poema en octavas sobre Il Nuovo mondo, publicado en 1628) le sirve a Góngora para modernizar el suyo. Por lo que, más que preguntarse quién canta en su fábula, cabría preguntarse si no es precisamente la alternancia de voces que en ella se enfrentan y se confunden su característica más peculiar y novedosa.

Quoi qu'il en soit, voici Polyphème chargé d'introduire dans la fable gongorine une imitation moderne, contemporaine, et d'incruster dans la fable mythologique des détails surprenants, uchroniques. Que ces strophes soient moins réussies que les autres, comme le prétendent Dámaso Alonso et Guyler, ne relève que de l'appréciation personnelle, mais ce que la littéralité textuelle met en relief c'est non seulement la métamorphose de Polyphème amoureux en hôte attentif et généreux mais en véritable " poète " de naufrages modernes qui ne laissent pas d'anticiper, une fois encore, sur le début de la Soledad primera et sur le fragment épique des Navigations.

\section{Le Polyphème de Góngora imitateur lyrique de Góngora ? La DERNIÈRE MÉTAMORPHOSE}

Analysant les réécritures de la fuite de Galatée dans la poésie classique et chez les poètes du Quattrocento, Angelo Poliziano et les «néo-latins » Pontano, Sannazaro et Bembo, Mélanie Bost-Fiévet propose, dès son introduction ${ }^{30}$ l'hypothèse suivante : "Polyphème devient un avatar du poète, décliné en figures variées, condamné à chanter la remémoration littéraire d'une fuite, à répéter pour les faire siennes les plaintes musicales des poètes qui l'ont précédé [...] ». Elle reprend la formule plus loin : «Les textes des néo-latins suggèrent un Polyphème qui devient progressivement image du poète ", et l'étaye, textes à l'appui, par la belle Lyra XVI de Pontano, où confluent la rhétorique de l'« ubi sunt » et l'énumération des pouvoirs d'Orphée ${ }^{31}$ :

Repris, légèrement modifié et traduit en italien, cet article, réintitulé "Mi voz por dulce, cuando no por mía..." ", est republié dans : Giulia Poggi, Gli occhi del pavone. Quindici studi su Góngora, Firenze, Alinea editrice, 2009, p. 187-206. Tout récemment, une version considérablement résumée et remaniée, "¿ Quién canta en el Polifemo ? Huellas de Stigliani en la fábula gongorina ", a été publiée dans Ínsula 781-782, loc. cit., p. 10-15 et, pour la citation : p. 14-15.

29. Samuel L. Guyler, "Góngora's Polifemo : the Humour of Imitation ", Revista Hispánica Moderna, XXXVII, 1972-1973, p. 237-252.

30. Mélanie Bost-Fiévet, "Cur fugis ... ?", art. cit., p. 4.

31. Ibid., p. 15-16 et, pour le texte latin et traduit, p. 26. 
Mais quoi ! est-ce là le Polyphème d'autrefois, celui-là, / Qui pleure devant les flots ? Hélas! Quelle honte / Pour la flûte mélodieuse qui jadis émouvait / Par sa douceur les tilleuls et les aulnes, / Au chant de laquelle accouraient le bétail, / Les bêtes sauvages sorties de leurs tanières, les oiseaux / Descendus du ciel, et du lointain rivage de Palica, / Les grenouilles muettes. Au son de mon chalumeau, la délicate Gélaé, / Au son de ma flûte, la douce Lilybée, / Au son de mes roseaux, la blonde Acianée / Se hâtaient de me rejoindre, / Et souvent à nos chants Aréthuse / Se laissa fléchir et fit danser ses doux chœurs, / Souvent à notre musique Anapé / Vint jouer sur la plage (v. 5-20).

Le Lycon de Sannazaro (Eclogae piscatoriae II) revisite aussi le discours du Cyclope « en laissant entendre, dans ses mots, la présence d'une "persona» de poète » :

Pourquoi t'enfuir ? On prépare pour toi des laines à la teinture, / Par elles ton éclat surpassera, Galatée, les autres jeunes filles / - de la laine plus douce que l'écume de la mer. C'est le berger / lui-même qui jadis me l'offrit, le berger Méliséus, un jour / que le vieil homme m'avait entendu chanter du haut d'une falaise, / me disant : "Jeune homme, que ces présents récompensent tes Muses, / puisque le premier tu as chanté sur nos rives." / Ce cadeau, je l'ai gardé dans des corbeilles, pour pouvoir te l'adresser ${ }^{32}$.

Dans l'article qu'elle consacre à l'évolution des représentations du mythe de Polyphème et à la " pulsion scopique » qui la justifie ${ }^{33}$, Mercedes Blanco évoque à deux reprises au moins la métamorphose de «l'ogre » en poète : ¿¿Qué extraña asociación de ideas ha hecho que el ogro se volviera encarnación del enamorado, y del poeta en calidad de enamorado [...]? » et, à propos des Métamorphoses d'Ovide : "En primer lugar no es ya este cíclope la mera máscara pastoril y satírica del poeta enamorado, sino el héroe de un drama".

Les rapports que je me propose d'analyser ici sont d'un tout autre ordre : il ne s'agit plus de considérer Polyphème amoureux comme double ou figure pastorale et satirique du poète mais de considérer qu'entre le chant du Polyphème de Góngora et la partie de la fable prise en charge par le poète narrateur s'établit, à propos de strophes bien précises, un lien de véritable réécriture. Je ne retiendrai ici que les strophes 7, 49 et 50 du portrait et de l'autoportrait du Cyclope, et les strophes 13 et 14 puis 46 du portrait de Galatée, mais il me semble que la comparaison devrait être menée à propos d'autres octaves du poème et de leur reprise par Polyphème. Il n'est pas douteux, en effet, que le défi majeur qu'avait à relever Góngora, dans son entreprise de réécriture de la matière polyphémique, était bien le chant du Cyclope, partout et toujours privilégié comme morceau de bravoure ou pierre de touche et moment exceptionnel de la fable mythologique. Par ailleurs, entre ce chant lyrique et bucolique de séduction et de plainte amoureuse et l'être monstrueux et terrifiant qui l'adresse à Galatée, la relation est pour le moins tendue et paradoxale : comment l'énorme Cyclope à la voix tonitruante qui fait fuir les vaisseaux et horrifie à ce point Triton qu'il casse sa trompe marine, peut-il vraisemblablement chanter

32. Ibid., p. 28.

33. M. Blanco, "Del Polifemo griego al barroco : un mito y sus imágenes ", in Ínsula 781 782, loc. cit., p. 3-6 et, pour les citations, p. 4-5. 
une cantilène amoureuse ? Comment marier la "terribilità " du personnage avec la douceur bucolique du lyrisme amoureux ? La solution est difficile : ou bien sa cantilène est grossière comme lui et parfaitement comique ; ou elle est partiellement élégante et partiellement grotesque, le naturel cyclopéen revenant au galop après l'épanchement lyrique, ou elle est tout simplement élégante. Mais même dans ce cas, l'image du monstre peut interférer et infléchir la lecture critique vers une interprétation humoristique ou amusée de ses accents lyriques. La solution trouvée par Góngora est, on le verra, originale car elle suggère, à propos d'une question bien précise de ponctuation, que Polyphème et tout ce qui le concerne soient envisagés sous l'espèce d'un " composé instable » en quoi la littérature a figé le monstre mythologique.

Dans les octaves 49 et 50 de son chant à Galatée, Polyphème évoque, suivant ainsi la tradition mais en sélectionnant drastiquement les motifs, l'hyperbolique abondance de ses troupeaux ${ }^{34}$ puis, en lien avec les Géorgiques et l'Éneide virgiliennes ${ }^{35}$ les réserves de miel sauvage que l'abeille toujours en mouvement, industrieuse et ingénieuse "liba inquïeta, ingenïosa labra " (50.4). C'est ensuite son lignage prestigieux qu'il invoque pour séduire la nymphe, se targuant d'être le fils du "Jupiter marin ", (alors que le Polyphème d'Ovide affirme qu'il méprise Jupiter, le ciel et la foudre et ne tremble que devant Galatée), puis sa très imposante taille (52) et enfin l'œil solaire de son front (53) :

52

$$
\begin{aligned}
& \text { Sentado, a la alta palma no perdona } \\
& \text { su dulce fruto mi robusta mano; } \\
& \text { en pie, sombra capaz es mi persona } \\
& \text { de innumerables cabras el verano. } \\
& \text { ¿Qué mucho, si de nubes se corona } \\
& \text { por igualarme la montaña en vano, } \\
& \text { y en los cielos desde esta roca puedo } \\
& \text { escribir mis desdichas en el aire? }
\end{aligned}
$$

$$
\begin{aligned}
& \text { Maritimo alción roca eminente } \\
& \text { sobre sus huevos coronaba el día } \\
& \text { que espejo de zafiro fue luciente } \\
& \text { la playa azul de la persona mía: } \\
& \text { miréme y lucir vi un sol en mi frente } \\
& \text { cuando en el cielo un ojo se veía; } \\
& \text { neutra el agua dudaba a cuál fe preste, } \\
& \text { o al cielo humano o al cíclope terrestre. }
\end{aligned}
$$

Cet autoportrait en deux tableaux met en place les traits exceptionnels de la prosopopée polyphémique : la démesure de sa taille et le soleil de son œil unique qu'il contemple dans le miroir d'une mer calme. Or, Góngora avait déjà offert une description du géant dans les vers de l'octave 7 : 
Dans la strophe suivante (8. 6) Polyphème, aux cheveux noirs et à la barbe torrentielle devenait "este Pirineo ». Entre la somptueuse strophe 7 et les octaves 52 et 53 se tissent, d'un bout à l'autre du poème, divers échos de ressemblance et de dissemblance, comme si Polyphème réécrivait en l'amplifiant ou " imitait " la source gongorine de son autoportrait, ou plutôt comme si Góngora lui accordait le privilège inouï d'être le porte-parole chargé de dénouer et de mettre à plat, dans l'espace de deux octaves, l'extraordinaire et hyperbolique concentration de la strophe source. Les seuls quatre premiers vers de l'octave 7 , en effet, disent à la fois la taille et le grandiose oeil solaire du Cyclope, alors qu'il ne faut pas moins à Polyphème de deux strophes pour réécrire ces deux traits caractéristiques de son portrait. On observe aussi qu'il n'évoque pas son incroyable force et qu'il ne reprend pas le motif du pin transformé en bâton de berger ni du jonc flexible devenu élégante canne de la fin de l'octave. Mais ses deux strophes font bien autre chose que développer les quatre premiers vers de la septième. Les quatre vers initiaux de la strophe 52 regroupent, autour du motif de la taille, des mots qui appartiennent à d'autres moments et à d'autres éléments de l'évocation que fait Góngora du Cyclope et de la féconde Sicile : "dulce fruto " et l'adjectif " capaz " font écho à la description de la besace de Polyphème, v. 73-74: "Cercado es, cuanto más capaz más lleno, I de la fruta, el zurrón, casi abortada"; le fruit sucré de la palme (que le géant ajoute à la liste des six noms de fruits des strophes 10 et $11:$ : la serba, la pera, la castaña, el membrillo, la manzana, y de la encina el tributo ") rappelle la couleur du coing du vers 11. 82: «y, entre el membrillo o verde o datilado ; no perdona " rappelle la strophe 18. 142 : " a sus campañas Ceres no perdona "; l'adjectif "robusta" dans "mi robusta mano" résonne encore de son emploi immédiatement antérieur dans 51.6-7 : "que tanto esposo admira la ribera cual otro no vio Febo más robusto " et de l'écho beaucoup plus lointain de la Dedicatoria (3. 1-2) : "Treguas al ejercicio sean robusto / ocio atento, silencio dulce ", où on retrouve, comme dans l'octave 52, l'association "dulce " I "robusto»; enfin, les « innumerables cabras» renvoient, pour l'hyperbole du nombre, à la sixième strophe du poème (6. 45-48) et à la caverne-bergerie : « $y$ redil espacioso donde encierra / cuanto las cumbres ásperas, cabrio, / de los montes esconde : copia bella / que un silbo junta y un peñasco sella" et pour l'ombre gigantesque qui protège les innombrables chèvres du soleil, comme l'indique Vilanova, à Stigliani ${ }^{36}$.

36. A. Vilanova, op. cit., II, p. 577 : il s'agit de l'octave XVIII dans les Stanze pastorali du 
La précision, la simplicité et l'organisation rigoureuse des quatre distiques de cette strophe ont attiré l'attention des commentateurs : Jesús Ponce ${ }^{37}$ remarque que Polyphème s'évoque "sentado, en pie, (en pie) desde esta roca " et il cite l'éloge enthousiaste de Pedro de Valencia (mai 1613) qui recommande au poète d'adopter ce style fluide et naturel et de renoncer à l'obscurité de "lo intrincado y trastocado y extrañado": "Es verdad que sabe vuestra merced decir alta y grandiosamente, con sencillez y claridad, con breves periodos y cada vocablo en su lugar, como si fuese en prosa. A sus propios ejemplos le remito: Sentado, a la alta palma [...]».

Dans la strophe suivante, qu'on ne saurait pour autant assimiler à de la prose, Polyphème réussit le tour de force de dénouer le vers 7. 3, "développant " comme le ferait un mathématicien poète toutes les potentialités enfermées dans la formule resserrée : "de un ojo ilustra el orbe de su frente ». Pour ce faire, il a besoin du miroir de saphir de la mer, qui ne lui renvoie pas - comme c'est le cas dans d'autres poèmes polyphémiques - un reflet flatteur de son visage (Góngora rejette absolument tous les indices qui pourraient faire penser que son Polyphème se trouve beau). La structure en miroir, symétrique, a son analogue en rhétorique, la figure de l'hypallage, qui sert ici la parfaite adéquation des hyperboles au gigantisme du Cyclope : "miréme y lucir vi un sol en mi frente I cuando en el cielo un ojo se veía; / neutra el agua dudaba a cuál fe preste, I o al cielo humano o al cíclope terrestre ". Mais au-delà de la proposition d'une reformulation détendue d'images si denses qu'elles exigent une glose ou une réécriture, les deux derniers vers mettent en ouvre la figure de la "dubitatio " par laquelle le miroir de l'eau, «neutre ", hésite entre deux solutions également inouïes : il n'y a, dans le Polyphème gongorin, qu'un seul autre exemple de "dubitatio ", également appliqué à un portrait, celui de Galatée, à la strophe 14, où est peint avec tant de délicatesse son teint de lys et de roses que «duda el Amor cuál más su color sea, / o púrpura nevada, o nieve roja $"^{38}$.

Cependant, ce n'est pas seulement le teint mais les yeux, liés à son teint, de Galatée qui, dans l'octave 13, donnent lieu à l'élaboration la plus dense, la plus complexe, la plus originale de Góngora, avec, pour la première fois, semble-t-il, " ojos " employés comme métaphore de "estrellas " (alors que la métaphorisation inverse est si ancienne qu'elle en est comme lexicalisée) et l'emploi de "pluma » comme métaphore de son visage au teint éblouissant ${ }^{39}$ :

13

$$
\begin{aligned}
& \text { Son una y otra luminosa estrella } \\
& \text { lucientes ojos de su blanca pluma: } \\
& \text { si roca de cristal no es de Neptuno, } \\
& \text { pavón de Venus es, cisne de Juno. }
\end{aligned}
$$

Polifemo italien de Stigliani.

37. Jesús Ponce, ed. cit., p. 332-333.

38. Voir, dans le numéro d'Ínsula déjà cité, ma contribution : "Espejismos de la retórica ", p. $20-25$.

39. Ibid., p. 24-25. 
La réécriture par Polyphème de ce splendide fragment ajoute une fleur, les " claveles » aux roses et aux lys de la strophe 14, puis explique, c'est-à-dire déplie ou déploie ou développe en cinq vers les deux premiers hendécasyllabes de l'octave 13 :

46

\author{
¡Oh bella Galatea, más suave \\ que los claveles que troncó la Aurora; \\ blanca más que las plumas de aquel ave \\ que dulce muere y en las aguas mora; \\ igual en pompa al pájaro que grave \\ su manto azul de tantos ojos dora \\ cuantas el celestial zafiro estrellas! \\ ¡Oh, tú, que en dos incluyes las más bellas!
}

Il suffit à Polyphème de remplacer par des comparaisons les métaphores ou les identités de l'octave 13 (sans pour autant renoncer à d'autres figures), il lui suffit de montrer que " ojos » est la métaphore figée, lexicalisée qui sert à désigner "littéralement " les taches qui ornent le plumage du paon, et il lui suffit encore de nommer les étoiles célestes par leur nom " estrellas " pour que les difficultés se dénouent et que l'écriture prenne un tour différent, qui annonce déjà les voies nouvelles que la poésie ultérieure explorera.

La douceur, la suavité, caractérisent aussi bien la Galatée de Góngora que celle qu'il prête au chant de Polyphème, dont les quelques exemples cités montrent qu'il n'est pas, non plus, - contrairement à ce qu'écrit Giulia Poggi ${ }^{40}$ - dépourvu de douceur et de beauté, bien que la caractérisation de la voix du Cyclope par le poète passe toujours par des termes disant l'horreur et la violence tonitruante : "bárbaro ruido ", "horrenda voz", au son desquels "la selva se confunde, el mar se altera, I rompe tritón su caracol torcido, I sordo huye el bajel a vela y remo, I ¡tal la música es de Polifemo!" (12). Cependant, le Polyphème amoureux de Góngora sait aussi, par la douceur de son instrument calmer les flots démontés (même si c'est lui qui le dit, mais l'amour ne l'a-t-il pas rendu poète ?), il sait défaire les nœuds d'une écriture dense et difficile par une réécriture souplement développée et surtout il est, me semble-t-il, le seul à juger dissonante la musique au son de laquelle Galatée tresse des mouvements de danse avec ses compagnes :

48

$$
\begin{aligned}
& \text { Sorda hija del mar, cuyas orejas } \\
& \text { a mis gemidos son rocas al viento: } \\
& \text { o dormida te hurten a mis quejas } \\
& \text { purpúreos troncos de corales ciento, } \\
& \text { o al disonante número de almejas } \\
& \text { (marino, si agradable no, instrumento) } \\
& \text { coros tejiendo estés, escucha un día } \\
& \text { mi voz por dulce, cuando no por mía. }
\end{aligned}
$$

40. Insula, art. cit., p. 12 et 14. Giulia Poggi montre remarquablement comment Góngora a su mettre à profit quelques inventions de Stigliani (la surdité de Galatée à ses accents, par exemple) pour réélaborer le motif de la voix de Polyphème ignoré par Ovide et effleuré par Théocrite. 
Si la strophe convoque les échos d'Ovide, Garcilaso, Tansillo, Sannazaro, Marino, etc., elle condense aussi spectaculairement les cinq octaves gongorines (15 à 17, puis 20 et 21), consacrées au dédain et à la froideur que la nymphe réserve à tous ceux qui, brûlant d'un impossible amour pour elle, lui vouent un culte auquel elle reste insensible, mais qui ne parlent ni ne chantent, pas plus que ne parlera ni ne chantera son séducteur aimé, Acis. Elle lie également la douce Galatée à un univers musical dissonant et désagréable qui la rapproche peut-être de l'assourdissante disharmonie polyphémique. L'extraordinaire chute de l'octave : "escucha un día / mi voz por dulce, cuando no por mía", intelligemment analysée par G. Poggi, confirme, avec la virgule placée après "por dulce ", l'hypothèse de l'exégète italienne, à savoir que Polyphème a de sa voix une perception bien différente de celle qu'en ont les habitants de l'île et surtout de celle qu'en propose le "narrateur omniscient ". Polyphème en appelle donc à l'oreille musicale, et non sentimentale, de Galatée, s'effaçant totalement au bénéfice de son chant, dans une annulation pathétique de sa personne (démentie néanmoins par les strophes qui suivent). Dans ce cas, le Cyclope devient un composé instable de vanité ou de lucidité poétiques et d'apparent renoncement à soi.

Une autre possibilité, cependant, existe, celle de ponctuer en plaçant la virgule après "cuando no": "escucha un día / mi voz por dulce cuando no, por mía ». C’est celle que choisit Antonio Carreira, dans son édition des poèmes de Góngora publiée par la Biblioteca Castro ${ }^{41}$. Dans ce cas, la reconnaissance par le Cyclope de la rudesse et de la disharmonie de sa voix lui restitue une lucidité que la version précédente semblait lui refuser, le met en accord avec l'adjectivation terrible que lui attribue le poète narrateur et en appelle à la compréhension de Galatée, elle aussi immergée dans une musique dissonante. Le possessif "mía ", placé en fin de vers, et opposé à "dulce cuando no ", propulse sa personne au premier rang de ses qualités séductrices - que développent les strophes suivantes, "Pastor soy..., Del Júpiter soy..., Polifemo te llama..., tanto esposo..., mi robusta mano; mi persona..., cielo humano / cíclope celeste », etc. Il en résulte un autre composé instable de lucidité ou de modestie et de pathétique ou insupportable étalage de qualités, associé à une totale ignorance de l'union amoureuse d'Acis et de Galatée.

Dans les deux cas, Polyphème s'est transformé en une structure " métamorphique ", apte à basculer du côté du grotesque comme du pathétique, de la laideur comme de la beauté, de la caricature comme de l'authenticité, de la parodie grossière comme d'une poésie nouvelle, aussi sublime que celle que son chant réécrit en la transformant : c'est ce que montrent clairement, par exemple, le destin largement burlesque de la fable de Polyphème et Galatée dans la littérature espagnole postérieure à 1613 et, en contrepartie, les beautés du chant de Polyphème répété par Ulysse dans La Circe de Lope (1624), dans lequel sont considérablement renouvelées les comparaisons entre Galatée et les éléments de la nature, et où le couple des rivaux amoureux de la nymphe, le Cyclope

41. Ed. cit., p. 348. 
lui-même et Acis, se métamorphose en l'image contrastée de l'amant puissant, mûr et viril et du jeunot, gringalet et efféminé dont la voix de grillon et la lyre grinçante (celle de Góngora ?) sont loin de pouvoir se mesurer à l'ampleur du chant de Polyphème (et de Lope ?) :

v. 257

Si canta ese rapaz, sutil parece

su voz de grillo negro en verde trigo;

la lira que le adorna y desvanece,

sierra en nogal, tan desigual conmigo;

mi voz los altos montes estremece,

$y$ asombra el mar, de mi dolor testigo,

donde me escuchan, con sus ninfas bellas,

los peces igualmente y las estrellas.

v. 265

Querer con mi grandeza y hermosura
sus partes competir afeminadas,
era igualar al sol la sombra escura,
supuesto que de mí jamás te agradas.
Diga el cristal de aquesta fuente pura,
cuando estaban las aguas sosegadas,
si pudiera ser yo con poco aviso
más disculpado que lo fue Narciso.

En dépit du caractère démesuré de l'hyperbolique comparaison avec le beau Narcisse, qui pourrait rendre risible l'auto-éloge, la lecture du Deuxième chant de La Circe ${ }^{42}$, du moins dans sa première partie, consacrée aux " amores de Polifemo y Galatea " (et non aux amours d'Acis et Galatée), montre que jamais le Cyclope n'y est qualifié par l'horreur qu'il peut inspirer, tout au plus par la stupeur et la crainte que produit le spectacle de son incroyable taille. La montagne a beau faire pousser les arbres les plus hauts qui couronnent son sommet, Polyphème arrive à les dépasser : "puesto que se ve proporcionado / la frente mide con su verde extremo" (v. 117-118). Bien proportionné, barbu et chevelu comme il convient à son extraordinaire puissance, le géant amoureux chante, en des vers rustiques, de douces et tendres plaintes d'amour. Seule Galatée, après la mort d'Acis, l'appellera "fiero monstro " en reliant sa cruauté aux «celos ", la jalousie amoureuse. La mort d'Acis fait, certes, le lien avec l'épisode où l'astucieux Ulysse, horrifié par sa violence cannibale, réussit à le rendre aveugle, mais le monstre amoureux n'intériorise pas dans les mots du poème de Lope, comme il le fait dans ceux de Góngora, l'instable et permanente union des contraires qui en fait une exceptionnelle structure métamorphique capable de permettre, dans le cas d'un vers exceptionnel, une double ponctuation et une double lecture.

42. Lope de Vega, La Circe, en Obras poéticas, Edición, introducción y notas de José Manuel Blecua, Barcelona, Planeta, 1983, Canto segundo, 973-999 et, pour les citations, 980-981. Dans la deuxième partie de ce deuxième chant (985-999), Polyphème redevient le monstre violent et sanguinaire que l'astuce d'Ulysse réussira à rendre aveugle et impuissant. 